\title{
Formation et transformation de défauts ponctuels par insolation UV dans les diélectriques à base de silice : application à l'intégration de composants optiques sur fibre
}

\author{
A. Boukenter, F. Goutaland, H. Kuswanto, E. Marin, J.P. Meunier et Y. Ouerdane \\ Université de Saint-Étienne, Laboratoire de Traitement du Signal et Instrumentation, \\ UMR 5516 du CNRS, 23 rue du Dr. Michelon, 42023 Saint-Étienne cedex 02, France
}

\begin{abstract}
Résumé: La présence dans les verres germanosilicates de défauts ponctuels résultants de défauts de liaisons interatomiques ou de stoechiométrie peut être à l'origine d'une modification photoinduite sous insolation UV de leur indice de réfraction. Cette modification est mise à profit pour induire une modulation périodique ou variable de l'indice permettant la réalisation de composants optiques intégrés sur fibre : filtres, miroirs, compensateurs de dispersion chromatique, étireur-compresseur d'impulsion optique...
\end{abstract}

\section{INTRODUCTION}

Les premières études portant sur les défauts ponctuels dans les diélectriques vitreux et plus particulièrement dans la silice datent des années cinquante [1]. Longtemps dédiées à la détermination de la nature des défauts et aux effets du rayonnement énergétique (X, $\gamma$, neutrons...) sur les diélectriques, leur intérêt s'est porté par la suite, et surtout avec l'avènement des transmissions optiques, sur l'implication de ces défauts dans l'atténuation optique. On a ainsi pu identifier et caractériser plusieurs types de défauts et les pertes qui leur sont associées. Les différents efforts ont abouti dans les années soixante-dix à la réalisation de fibres optiques à faibles pertes permettant des transmissions sans réamplification sur plusieurs dizaines de kilomètres. Pendant cette période, différents procédés d'élaboration, aussi bien des matériaux que des fibres, ont vu le jour et ont permis de limiter de manière significative la formation des défauts. Les années quatre-vingt ont été marquées par un changement du statut des défauts. De nuisibles et indésirables, certains défauts sont devenus prisés. A l'origine de ce revirement on trouve la découverte par K. O. Hill [2] du caractère photosensible des verres germanosilicates lié à la présence des défauts. La possibilité d'induire une variation permanente de l'indice de réfraction par insolation des guides optiques et son exploitation pour photoinscrire des réseaux de phase a valu aux défauts leur consécration. Cette découverte a ouvert très vite la voie à la réalisation de différents composants optiques intégrés sur guide: filtres, miroirs, résonateurs, compensateurs de dispersion chromatique...

Malgré l'industrialisation à l'heure actuelle de ces composants, l'origine et la structure précises des défauts impliqués dans la photosensibilité ainsi que les mécanismes de leurs formation et transformation ne sont pas encore complètement déterminés. Cette carence est liée à la nature du milieu hôte, désordonné et permettant un très grand nombre de configurations structurales. Les défauts dans ce milieu présentent de surcroît une forte dépendance des conditions d'élaboration du matériau et des traitements subis. 


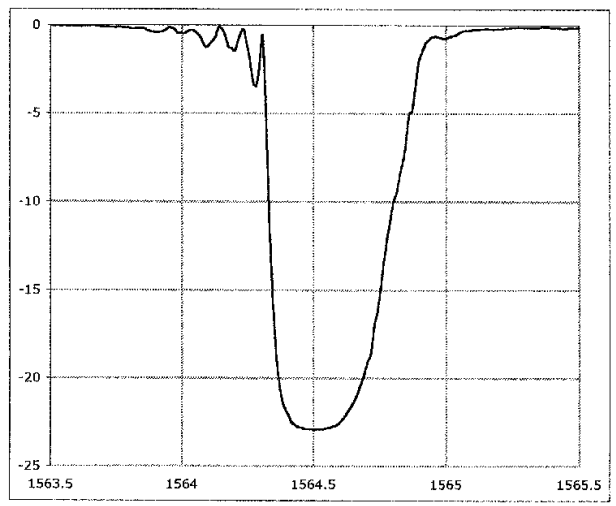

Figure 1: Spectre en transmission d'un réseau de Bragg uniforme

Pour réaliser un RB sur fibre optique, on expose la fibre à un champ d'interférences de quelques millimètres à quelques centimètres d'étendue. Les longueurs d'onde habituellement utilisées pour photoinscrire les RB se situent dans l'UV est correspondent à des bandes d'absorption de défauts ponctuels. Le champ d'interférences est obtenu soit par division du front d'onde, comme dans le cas du miroir de Lloyd, soit par division d'amplitude en utilisant généralement un masque de phase, figure 2 . Le premier montage, type miroir de Lloyd, permet une variation continue de la période $\Lambda$ mais n'est pas adapté pour la réalisation de réseau dépassant quelques millimètres de longueur. Les montages utilisant des masques de phase permettent, quant à eux, la réalisation de réseaux de plusieurs centimètres, typiquement 2 à $3 \mathrm{~cm}$, mais nécessitent l'utilisation d'un masque de phase pour chaque période $\Lambda$.

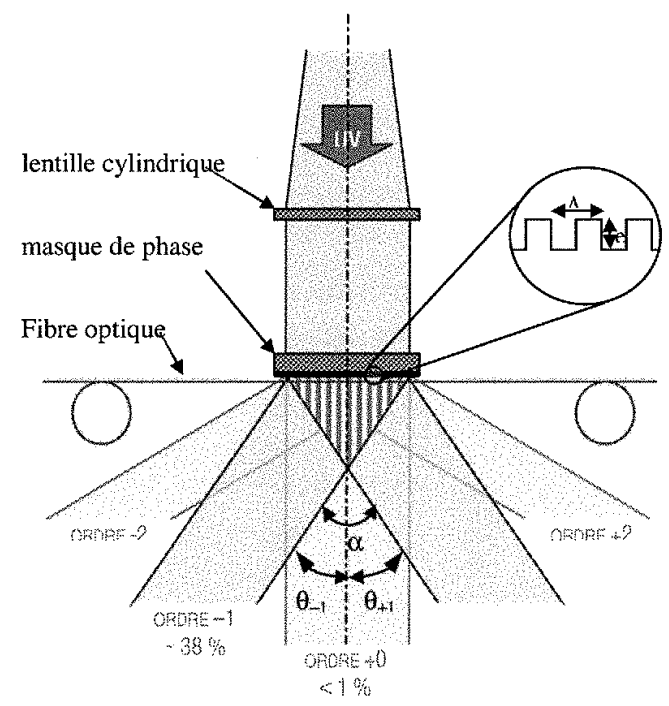

Figure 2 : Schéma du montage expérimental à masque de phase

\section{QUELQUES APPLICATIONS DES RÉSEAUX DE BRAGG SUR FIBRE OPTIQUE}

Les RB trouvent actuellement des applications dans l'ensemble des activités liées au traitement spectral de la lumière en optique guidée : télécommunications optiques, lasers, capteurs...où ils sont utilisés comme filtres, miroirs, compensateurs de dispersion chromatique, étireur - compresseur d'impulsion... 
Dans le domaine des télécommunications optiques la fonction de base des réseaux de Bragg consiste en l'insertion ou l'extraction d'un canal de transmission de longueur d'onde $\lambda_{i}$ parmi un ensemble de $n$ canaux, c'est le multiplexage en longueur d'onde (WDM). Le schéma de principe est donné dans la figure 3. L'espacement des canaux dans la fenêtre des télécommunications autour de $1.55 \mu$ peut atteindre $0.2 \mathrm{~nm}$ (25 GHz). Moyennant une centaine de canaux et à raison de $10 \mathrm{Gbits} / \mathrm{s}$ par canal, on peut obtenir sur une fibre unique un débit de transmission de quelques Tbits/s.

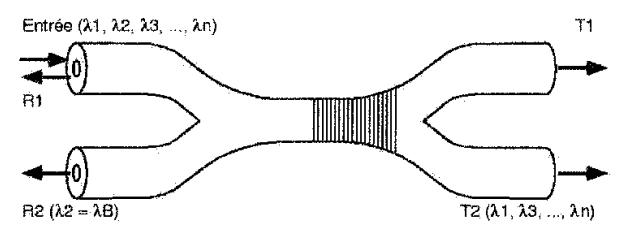

$3 . \mathrm{a}$

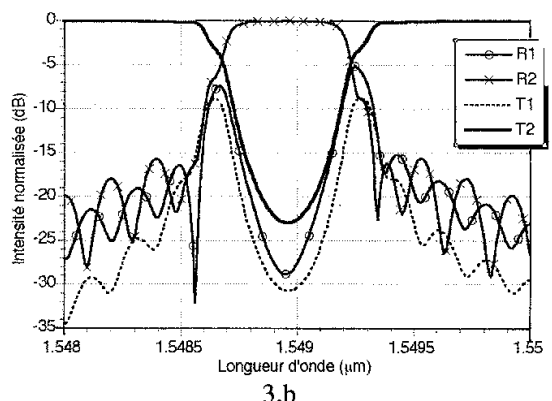

3.b

Figure 3: Schéma d'un multiplexeur à insertion/extraction (a) et sa réponse en longueur d'onde (b)

En plus du multiplexage, les RB sont utilisés dans les télécommunications optiques comme compensateurs de dispersion chromatique. Lors de la transmission des impulsions lumineuses et du fait de la dépendance en longueur d'onde de l'indice de la fibre, ces impulsions subissent un étalement temporel. L'utilisation d'un RB à pas variable permet de restaurer la forme des impulsions; les longueurs d'ondes ayant subi un retard sont réfléchies par la partie avant du RB alors que celles ayant la plus grande vitesse de groupe sont réfléchies par la partie arrière. Un tel RB constitue une ligne à retard chromatique. Ce même principe est aussi utilisé pour le traitement des impulsions lasers. Le RB peut compenser la dispersion chromatique ou jouer au contraire le rôle d'un étireur d'impulsion. La figure 4 ci-dessous donne la réponse temporelle d'un $\mathrm{RB}$ à période modulée.

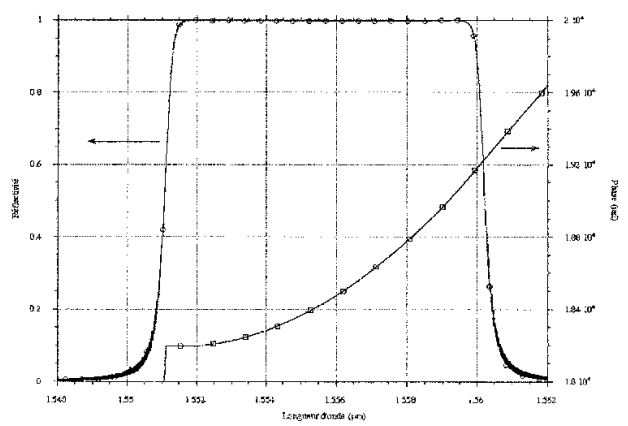

Figure 4 : Réflectivité et phase d'un réseau de Bragg à pas variable [8].

Dans le domaine des lasers fibrés, en plus de leur utilisation comme simple miroir de cavité, les RB sont aussi utilisés pour améliorer la platitude du gain. Dans ce cas, la réflexion du réseau est fonction de la longueur d'onde. Elle permet de favoriser certaines plages spectrales au détriment d'autres pour obtenir une puissance laser en sortie constante sur une large étendue spectrale [9].

Dans le domaine des capteurs, on exploite essentiellement la variation de la réponse spectrale des RB en fonction des sollicitations extérieures : température, contrainte, indice de réfraction du milieu extérieur... La modification de l'environnement du RB se reflète sur l'indice effectif ou la périodicité du réseau et affecte de ce fait la longueur d'onde de Bragg $\left(\lambda_{\mathrm{B}}=2 \Lambda \mathrm{n}_{\mathrm{eff}}\right)$. Pour les capteurs de température ou de déformation utilisant des RB photoinscrits sur des fibres standards, la variation de $\lambda_{B}$ est de l'ordre 


\section{DÉFAUTS PONCTUELS DANS LES VERRES GERMANOSILICATES}

Les verres germanosilicates constituent le matériau de base pour l'élaboration des fibres standards de télécommunications optiques. Dans ces verres les atomes de silicium et de germanium forment des liaisons covalentes avec quatre atomes d'oxygène arrangés en tétraèdre. Chaque tétraèdre est relié à quatre autres par des liaisons de type $\mathrm{T}-\mathrm{O}-\mathrm{T}(\mathrm{T}=\mathrm{Si}$ ou $\mathrm{Ge})$. Cet arrangement local est très similaire à celui de la forme cristalline. Les longueurs de liaisons et les angles sont très voisins dans le cristal et le verre. La rupture de l'ordre local dans la forme vitreuse se manifeste par l'apparition de défauts à l'échelle de la liaison entre atomes : les défauts ponctuels. Ces défauts sont soit des défauts de liaison ou de stœchiométrie.

Dans le premier cas on trouve essentiellement les défauts NBOHC "Non Bridging Oxygen Hole Centers", $\equiv \mathrm{T}-\mathrm{O} \cdot$, et $\mathrm{E}^{\prime}, \equiv \mathrm{T} \bullet$, résultant de la rupture de liaisons $\mathrm{T}-\mathrm{O}$. Les défauts de stœechiométrie sont, quant à eux, le fruit de la formation de liaisons 'contre nature' comme O-O, T-T, Si-Ge ou de liaisons multiples. Une revue de l'ensemble des défauts rencontrés dans les verres germanosilicates est donnée dans la référence [3].

Parmi les défauts des verres germanosilicates, ceux déficients en oxygène présentent un intérêt particulier pour la photosensibilité. Ces défauts possèdent souvent une ou plusieurs bandes d'absorptions dans l'UV entre 3 et $8 \mathrm{eV}$ et sont susceptibles d'être formés ou transformés sous insolation ultraviolette. Le photoblanchiment, par exemple, sous insolation UV des GODC, Germanium Oxygen Dificient Centers, contribue à la modification de l'indice de réfraction et est exploité pour intégrer des composants optiques sur fibre.

La variation d'indice photoinduite dans les fibres standard est de l'ordre de quelques $10^{-5}$ et est insuffisante pour la réalisation de certains composants. Pour obtenir des variations de l'ordre de $10^{-3}$, on utilise des fibres avec un fort pourcentage d'oxyde de germanium, $20 \%$ environ, ou on procède à un traitement préalable des fibres classiques. Ce traitement est basé le plus souvent sur une charge des fibres en hydrogène combinée ou non avec un traitement thermique et une préinsolation UV. Ce traitement présente cependant l'inconvénient de générer des défauts comme les hydroxyls, $\mathrm{OH}$, qui augmentent l'atténuation des fibres optiques dans les fenêtres de télécommunication optique. En plus de ces deux possibilités, de nouvelles compositions de fibres silicatées, utilisant notamment le plomb et l'azote comme dopants, ont vu dernièrement le jour et permettent d'obtenir des variations d'indice de l'ordre de $10^{-2}[4,5]$. La compréhension des mécanismes à l'origine de la variation de l'indice de réfraction $\Delta \mathrm{n}$ sous insolation UV est d'un grand intérêt pour l'optimisation des procédés de photoinscription de modulations d'indice. Deux modèles ont été proposés dans les années quatre-vingt-dix pour la détermination quantitative de $\Delta \mathrm{n}$ : Le modèle de centres colorés et le modèle mécanique.

Le premier, dû à Hand et Russell [6], relie le changement permanent de l'indice de réfraction aux modifications induites par insolation dans le spectre d'absorption. Ces dernières sont générées par la création et la destruction de différents défauts structuraux optiquement actifs, les centres colorés. Le second modèle, proposé par Poumellec et al. [7], exploite la densification et le changement des contraintes induits par insolation UV pour rendre compte de la variation d'indice, le changement de position des atomes lors du changement de configuration créant une nouvelle répartition des contraintes et un changement local de la densité du matériau.

\section{TECHNIQUES DE RÉALISATION DE RÉSEAUX DE BRAGG SUR FIBRE OPTIQUE}

Un réseau de Bragg (RB) est une modulation permanente de l'indice de réfraction de la fibre optique. Pour une modulation de période $\Lambda$, l'accord de phase est obtenu pour une onde électromagnétique de longueur d'onde $\lambda_{B}$ dite longueur d'onde de Bragg tel que $\lambda_{B}=2 \Lambda n_{\text {eff }}$ où $n_{\text {eff }}$ est l'indice effectif du mode fondamental de la fibre. Cette longueur d'onde est réfléchie par le réseau de Bragg alors que le reste de la lumière injectée dans la fibre est transmis. Le réseau de Bragg joue dans ce cas le rôle d'un miroir sélectif. La figure 1 donne la réponse spectrale par transmission d'un RB photoinscrit sur une fibre de télécommunications optiques standard. 
respectivement de $20 \mathrm{pm} /{ }^{\circ} \mathrm{C}$ et $1 \mathrm{pm} / \mu \mathrm{m} / \mathrm{m}$ [10]. Ces sensibilités permettent, avec les systèmes d'interrogation disponibles actuellement, des mesures de la température au dixième de degré et de la déformation au $\mu \mathrm{m} / \mathrm{m}$ près.

\section{CONCLUSION}

La présence des défauts ponctuels dans les verres germanosilicates leur confère des propriétés permettant la modification de leurs caractéristiques physiques. Sur le plan fondamental, l'étude des mécanismes microscopiques conduisant à la création et à la transformation de ces défauts, sous différentes sollicitations, est à la base de la compréhension de l'origine des modifications des propriétés macroscopiques de ces matériaux. Sur le plan des applications, la modification des propriétés du milieu, et plus particulièrement de l'indice de réfraction, est mise à profit pour la réalisation de composants optiques pour les télécommunications, les lasers et les capteurs.

\section{Réferences}

[1] Weeks R.A., J. Appl. Phys. 27 (1956) 1376-1381.

[2] Hill K.O., Fuji Y., Johnson D.C. et Kawasaki B.S., Appl. Phys. Lett. 32 (1978) 647-650.

[3] Kuswanto H., "Formation et transformation des défauts ponctuels dans les fibres optiques germanosilicates", Thèse de Doctorat. Université de Saint-Etienne (2001).

[4] Long X.C. et Brueck S.R.J., Appl. Phys. Lett. 74 (1999) 21 10-2112.

[5] Butov O.V., Golant K.M. et Nikolin I.V., Elect. Lett 28 (2002) 523-525.

[6] Hand D.P. et Russel P. St. J., Opt Lett., 15 (1990) 102-104.

[7] Poumellec B., Niay P., Douay M. et Bayon J.F., J. Phys. D: Appl. Phys., 29 (1996) 1842-1844.

[8] Marin E., "Modélisation des effets des réseaux de Bragg dans les composants à fibre optique" Thèse de Doctorat. Université de Saint-Etienne, (2000).

[9] Guillaumond D., "Etude théorique et expérimentale d'une source à fibre dopée Erbium pour un gyromètre à fibre optique hautes performances", Thèse de Doctorat. Université de Saint-Etienne (2000).

[10] Lepley D., "Intégration de capteurs à fibre optiques dans des isolateurs électriques à structure composite", Thèse de Doctorat. Université de Saint-Etienne (2001). 